\title{
Patterns of expression of murine Vgr-1 and BMP-2a RNA suggest that transforming growth factor- $\beta$-like genes coordinately regulate aspects of embryonic development
}

\author{
Karen M. Lyons, Ron W. Pelton, and Brigid L.M. Hogan \\ Department of Cell Biology, Vanderbilt University, Nashville, Tennessee 37232 USA
}

The murine Vgr-1 (Vg-related) and BMP-2a (bone morphogenetic protein 2a) genes are members of the decapentaplegic subgroup of the transforming growth factor- $\beta$ (TGFß) superfamily. Although genetic and biochemical studies suggest that the members of this subgroup play important roles in development, little is known about their function in mammals. Therefore, we investigated the expression of Vgr-1 and BMP-2a RNAs in embryonic, newborn, and adult tissues by in situ hybridization. Vgr-1 RNA is maternally encoded in ovarian oocytes but declines in fertilized eggs and is undetectable by the two- to four-cell stage. Only low levels of transcripts are seen in blastocysts and early postimplantation stages. From mid-gestation on, Vgr-1 RNA is expressed at high levels in developing skin, especially in the suprabasal cells of the proliferating epidermis but not in the dermis or hair follicles, both of which contain TGFß1 and/or TGFß2 RNAs. In contrast, BMP-2a transcripts are seen only in the hair follicles in the cells of the hair bulb cortex. Temporally and spatially distinct patterns of BMP-2a, Vgr-1, TGFß1, and TGFß2 expression are also seen in different populations of mesenchymal cells in the developing skeletal system (cartilage and bone). Our results suggest that the coordinated expression of several members of the TGF $\beta$ superfamily is required to control the progression of specific cell types through their differentiation pathways.

[Key Words: Vgr-1; BMP-2a; TGF $\beta$; in situ; development]

Received July 24, 1989; revised version accepted September 4, 1989.

The transforming growth factor- $\beta$ (TGF $\beta$ ) superfamily contains at least five gene products closely related to TGF $\beta 1$, as well as several more distantly related molecules such as Müllerian inhibiting substance (MIS), bone morphogenetic proteins, the Drosophila decapentaplegic (dpp) gene product, and the Xenopus Vg1 gene product. Numerous studies have indicated that members of both subgroups play important regulatory roles in growth and development. TGF $\beta 1$ and TGF $\beta 2$ have been isolated from a variety of adult tissues and can either stimulate or inhibit cell proliferation and differentiation and have positive or negative effects on specific gene expression in model systems in vitro (see, e.g., Moses et al. 1985; Roberts et al. 1985; Ignotz and Massagué 1986; Edwards et al. 1987; Lund et al. 1987; Coffey et al. 1988; D.M. Rosen et al. 1988; Russell et al. 1988). In addition, several in vivo studies have demonstrated that TGF $\beta 1$ affects the growth and differentiation of adult tissues (Roberts et al. 1986; Silberstein and Daniel 1987; Daniel et al. 1989), and immunohistochemical and in situ hybridization studies further support the notion that TGF $\beta 1$ and TGF $\beta 2$ are involved in regulating the morphogenesis of embryonic tissues (Heine et al. 1987;
Lehnert and Akhurst 1988; Sandberg et al. 1988; Flanders et al. 1989; Pelton et al. 1989; Thompson et al. 1989). Finally, the observations that porcine TGF $\beta 1$, in combination with basic fibroblast growth factor (bFGF), and porcine TGF $\beta 2$ alone can induce the expression of mesoderm specific markers in isolated Xenopus animal cap cells provide additional evidence that members of this subgroup regulate developmental events in vivo (Kimmelman and Kirschner 1987; Rosa et al. 1988).

Several members of the second subgroup, which show greater homology to the Drosophila dpp gene product than to TGF $\beta 1$, also have critical roles in embryogenesis. Drosophila embryos homozygous for null alleles of $d p p$ are completely ventralized (Irish and Gelbart 1987). Transcripts of the $d p p$ gene are detected in the embryo and larva, predominantly in the dorsal ectoderm and epithelial imaginal discs but also in the visceral mesoderm and gut endoderm, suggesting that the $d p p$ gene product participates in a number of morphogenetic events (St. Johnston and Gelbart 1987). The closely related bovine bone morphogenetic protein 2a (BMP-2a) induces ectopic cartilage formation in vivo, raising the possibility that this protein is involved in bone forma- 
tion and remodeling during mammalian embryogenesis (Wozney et al. 1988). The Vg1 gene product was isolated as a maternally encoded mRNA that becomes progressively localized to the vegetal hemisphere of Xenopus embryos and then declines in level after gastrulation (Rebagliati et al. 1985). Several different signals originating in the vegetal hemisphere are thought to induce mesoderm formation in the overlying animal pole cells in a complex process known as mesoderm induction (Dale and Slack 1987; for review, see Smith 1989). On the basis of its temporal and spatial expression, $\mathrm{Vgl}$ is a candidate for a mesoderm-inducing factor.

To study the potential roles of members of the $d p p$ subgroup during murine development, an 8.5-day postcoitum (p.c.) embryo cDNA library was screened with a partial cDNA from the Xenopus Vgl gene. A cDNA, designated Vgr-1 (for Vg-related) was isolated. The carboxyterminal portion of the predicted protein encoded by the Vgr- 1 cDNA shows $78 \%$ and $77 \%$ similarities to the corresponding regions of the Xenopus $\mathrm{Vgl}$ and Drosophila dpp gene products, but only $45 \%$ similarity to the corresponding portion of TGF $\beta 1$ (Lyons et al. 1989). Thus, Vgr-l belongs to the subgroup of $d p p$-like genes within the TGF $\beta$ superfamily.

Previous Northern analyses showed that Vgr-1 RNA is expressed at many stages throughout development in specific tissues. (Lyons et al. 1989). Here we examine the spatial distribution of these transcripts by in situ hybridization. We show that Vgr-l RNA is maternally encoded and is present in primary oocytes and fertilized eggs but is not detected in cleavage-stage embryos. In postimplantation embryos, Vgr-1 RNA is present in many of the same tissues shown previously to contain TGF $\beta 1$ and TGF $\beta 2$ transcripts but with a temporally and spatially distinct pattern of expression. Vgr-1 also has a different pattern of expression from BMP-2a, another member of the $d p p$ subfamily. These observations raise the possibility that the coordinated expression of different members of the TGF $\beta$ superfamily is required to regulate, by autocrine and paracrine feedback mechanisms, the progression of specific subpopulations of cells along specific differentiation pathways during mammalian development.

\section{Results}

\section{Expression of Vgr-1 RNA in oocytes and early embryos}

To characterize Vgr-1 expression at early stages of murine development, we localized RNA in oocytes, fertilized eggs, and pre- and postimplantation embryos by in situ hybridization. In the adult ovary, Vgr-1 RNA is present at high levels in both immature and mature oocytes (Fig. 1A,B). A much lower level of hybridization can be detected in the somatic follicle cells surrounding the developing oocytes. Northern analysis (Fig. 1E) shows that the Vgr-1 transcripts in oocytes and follicle cells are slightly larger than those detected in other cell types. Vgr-1 transcripts are present in fertilized eggs at a level somewhat lower than in oocytes (Fig. 1C,D). Quantization of the density of autoradiographic grains indicates that the fertilized eggs contain $50-70 \%$ the level of the Vgr-1 RNA present in ovarian oocytes with an intact germinal vesicle. Vgr-1 RNA is undetectable at the two- to four-cell stage (Fig. 1F,G), and the 8- to 16cell stage (data not shown) but a low level of signal is present (Fig. 1H,I) in 4.5-day p.c. blastocysts. Hybridization to the Vgr-1 riboprobe is also detectable in 6.5- to 12.5-day p.c. embryos, as judged by comparison of overall grain density in sections hybridized with sense and antisense RNA. However, there appears to be no specific localization to a particular germ layer or region (data not shown). Beginning at day 13.5 p.c., Vgr-1 transcripts could be detected in specific tissues, as discussed in the following sections.

\section{Expression of $V g r-1$ and BMP-2a RNAs in epithelia}

A preliminary survey of embryonic, newborn, and adult tissues showed that Vgr-l RNA is localized predominantly, but not exclusively, in stratified squamous epithelia. For example, it is present in the suprabasal layers of the developing skin (Fig. 2) and in adult cervix, vagina, and esophagus (data not shown). A particularly high level of Vgr-1 RNA expression is seen in the stratified squamous epithelium of the adult forestomach (Fig. $3 \mathrm{~A}-\mathrm{D} \mid$. Furthermore, a sharp discontinuity in Vgr-1 expression occurs within a few cells at the squamo-columnar junction between the forestomach and the gastric epithelium (Fig. 3E,F, arrowheads). Not all stratified squamous epithelia express Vgr-1 RNA at high levels, however, and a much lower level of hybridization, confined to the uppermost layers of the epithelium, is present in the developing palates, lip (Fig. 2G,H,K,L), and tongue (data not shown).

To investigate the possible roles of members of the $d p p$ subfamily in epidermal morphogenesis in more detail, we examined the expression of both Vgr-1 and BMP-2a transcripts in developing skin from 13.5 days p.c. to birth, and in adults. Vgr-1 expression is not seen at 13.5 days p.c. (Fig. $2 \mathrm{~A}, \mathrm{~B}$ ) but is first detected at 15.5 days p.c. within the thickening epidermis (Fig. $2 \mathrm{C}, \mathrm{D})$. Expression continues in the suprabasal layers throughout embryogenesis and in newborns (Fig. 2C,D, M-P). Neither the hair follicles nor the dermis show hybridization at any stage of development. Vgr-1 RNA could not be detected in adult dorsal epidermis (data not shown). This pattern of expression is in contrast to that of TGFß2 (Pelton et al. 1989) and BMP-2a in developing epidermis. As shown in Figure 2, E and F, TGF 22 RNA is first detected at 15.5 days p.c. within the dermis. At 17.5 days p.c., TGF $\beta 2$ expression in the dermis begins to decline, and by 18.5 days p.c., transcripts appear in the epidermis and in the outer root sheath of the hair follicles. This pattern of TGFß2 expression persists at least through 3 days postpartum (Pelton et al. 1989).

A very different pattern of expression is observed when in situ hybridization is carried out using a BMP-2a antisense probe. Expression is not seen at any stage in the interfollicular epidermis but is confined to developing hair and whisker follicles (Lyons et al. 1990; data 

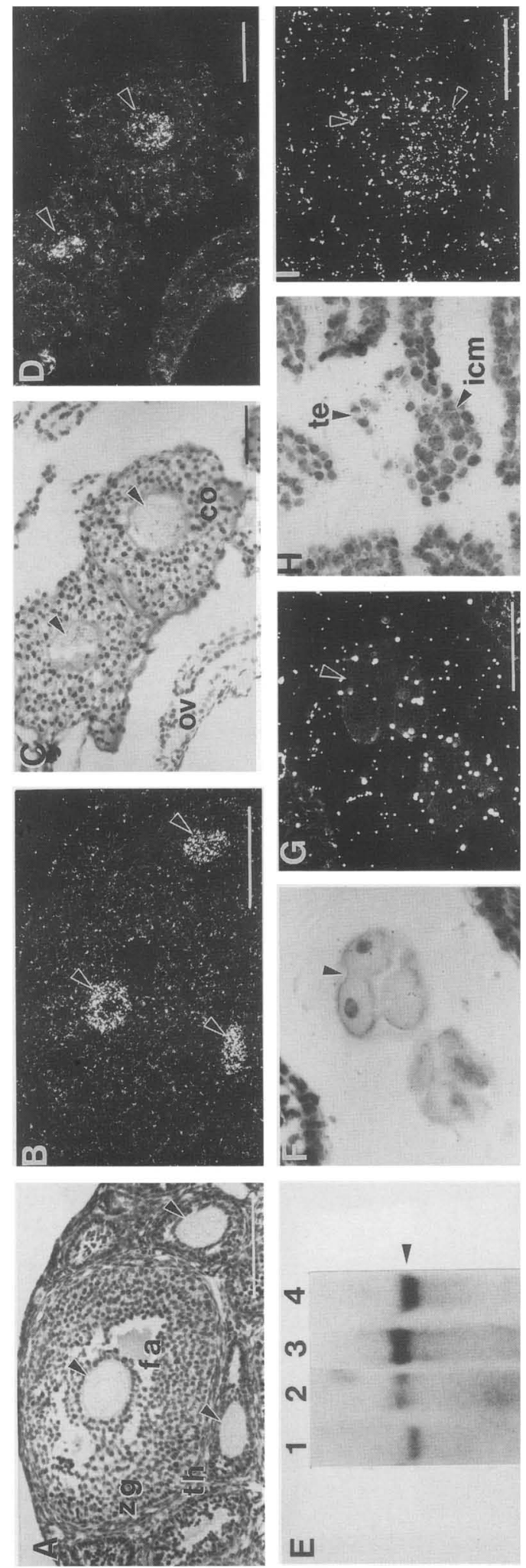

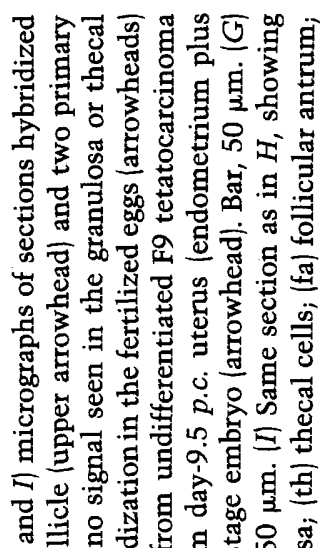

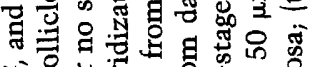

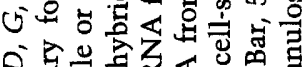

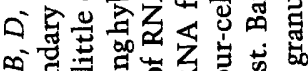

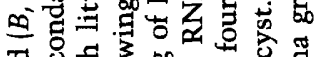

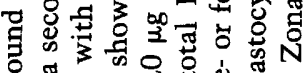

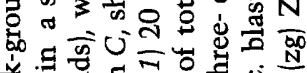

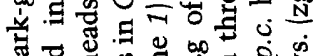

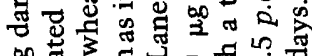

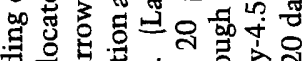

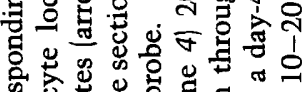

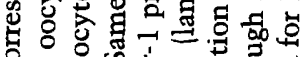

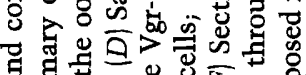

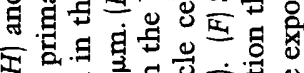

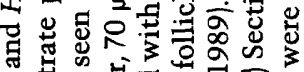

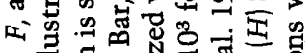
ט

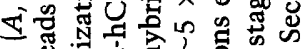

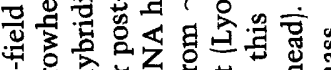

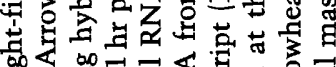

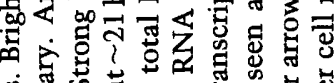

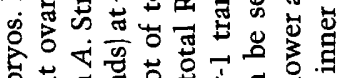

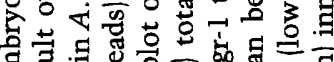

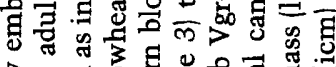

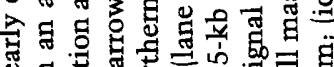
ङ5

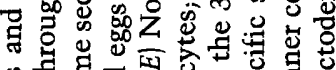

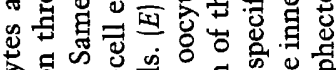

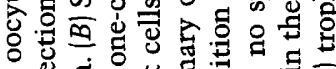

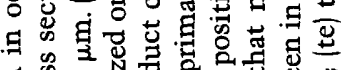

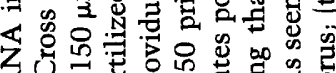

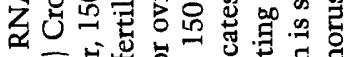

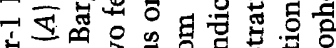

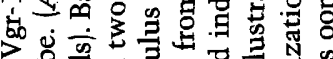

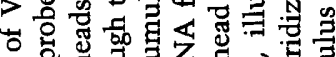

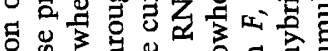

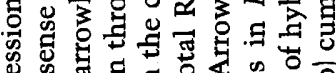

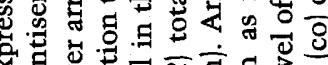

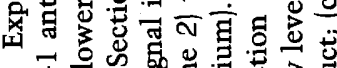

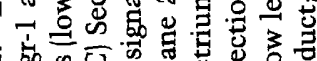

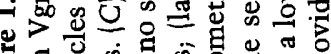

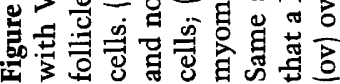


Lyons et al.
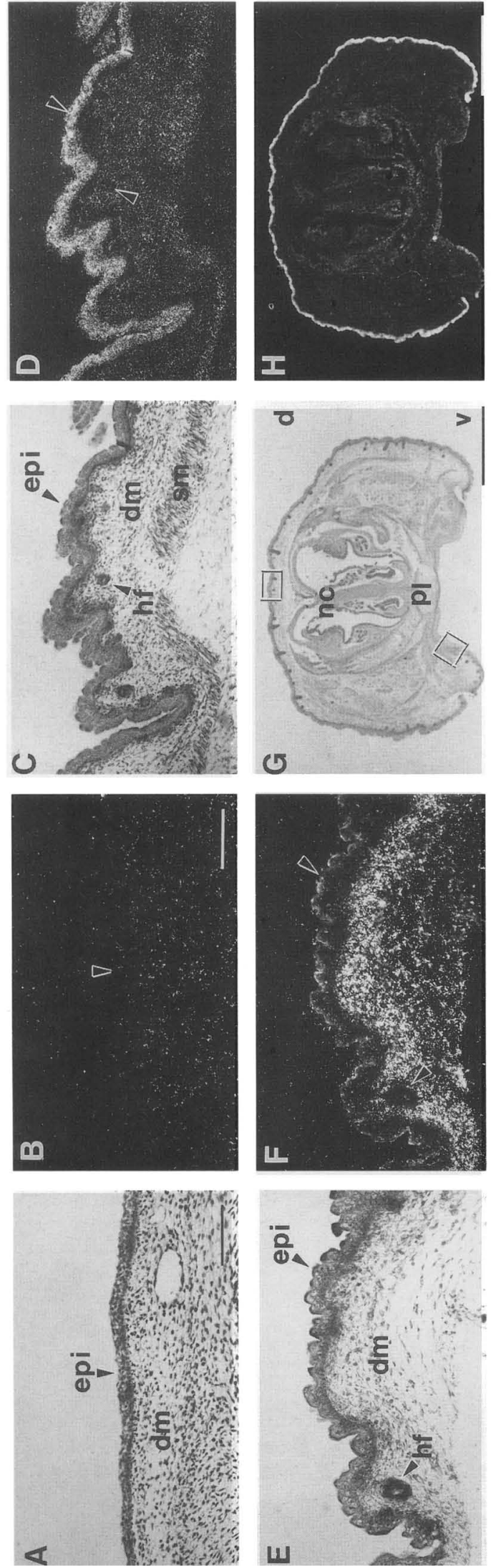
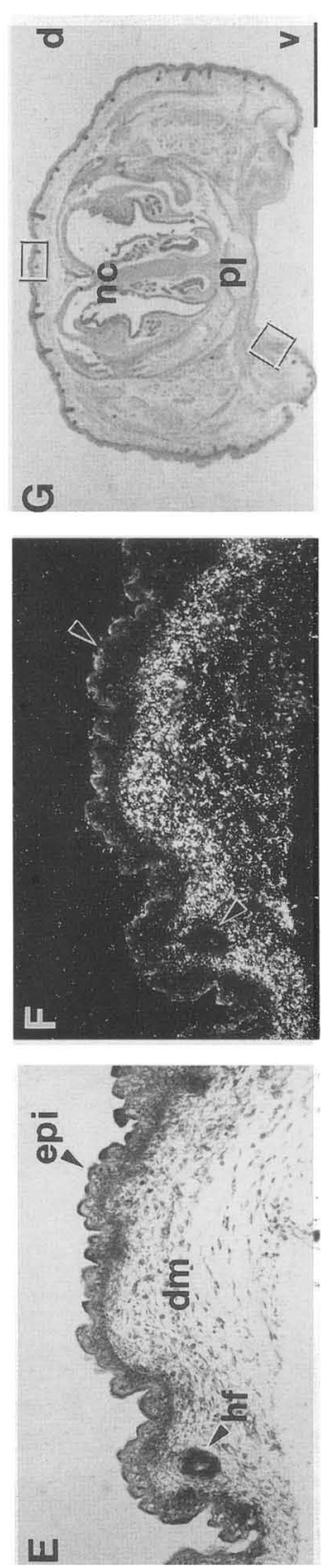
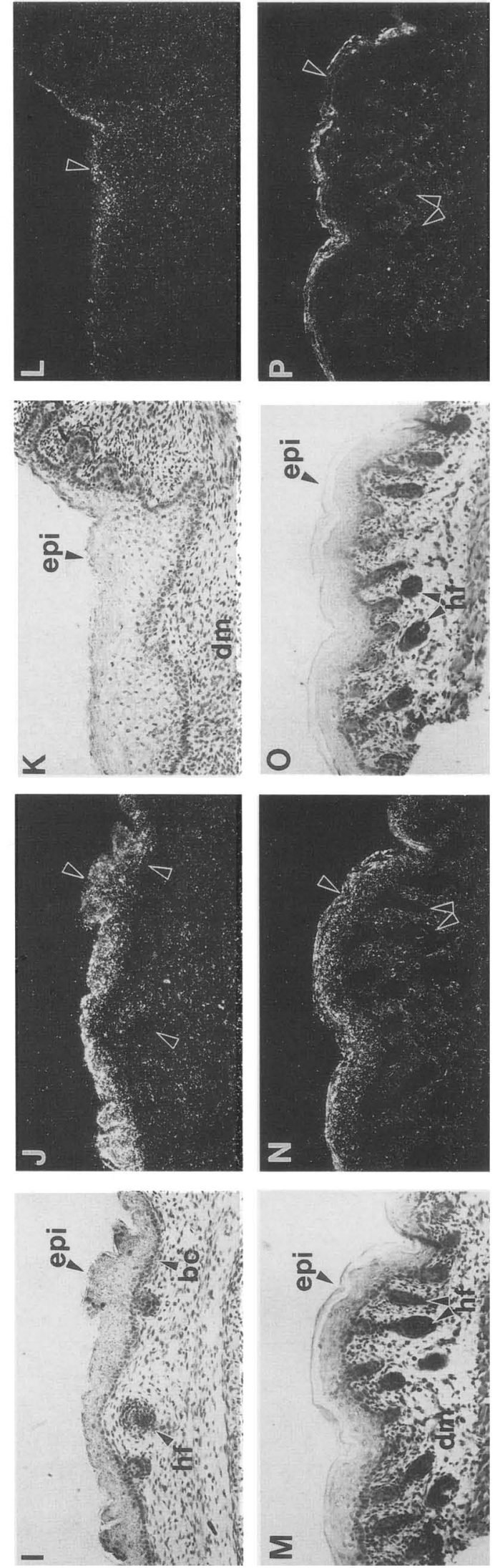
not shown). Expression in the hair follicles persists throughout adulthood and appears to be localized specifically to the cortex/inner root sheath of the hair shaft (Fig. 4).

\section{Expression of Vgr-1 RNA in mesenchymal tissues}

Vgr-1 transcripts are present in some tissues of mesodermal origin. In particular, high levels are seen in hypertrophic cartilage throughout the embryonic skeletal system, including the developing limbs, vertebral column, ribs, and head.

Examination of developing limbs from 11.5 to 18.5 days p.c. showed strikingly different patterns of localization for BMP-2a and Vgr-1. As reported previously (V. Rosen et al. 1988), BMP-2a RNA is present in the precartilaginous mesenchyme of the limbs. Figure 5, A and $\mathrm{B}$, shows that BMP-2a transcripts are localized in prevertebrae of 12.5-day p.c. embryos. Upon examination of 13.5- to 18.5-day p.c. developing limbs, a low level of BMP-2a expression is also present in the periosteum and osteogenic zone (data not shown). Vgr-1 RNA is first detected at 13.5 days p.c. and is specifically localized to the cells of the hypertrophic cartilage. This localization continues throughout development and in newborns (Fig. 5C-F). No Vgr-1 expression is seen in the perichondrium, periosteum, osteoblasts, osteoclasts, osteocytes, or tendon, all of which have been shown to contain TGF $\beta 1$ and/or TGF $\beta 2$ transcripts (Lehnert and Akhurst 1988; Sandberg et al. 1988; Pelton et al. 1989).

Expression of Vgr-1 is also seen in developing and adult meninges. No expression is apparent in the meninges of 12.5-day p.c. embryos, which do not contain a distinct arachnoid layer at this stage. In the newborn and in adults, Vgr-1 transcripts are abundant in all three layers of the meninges surrounding the brain and spinal cord (data not shown).

Vgr-1 transcripts are also detected in the stromal component of the uterine endometrium in adult female mice (data not shown). Uterine expression of Vgr-1 may be regulated during the estrous cycle, because no Vgr-1 RNA is seen in some female mice. However, we have not attempted to correlate the level of Vgr-1 expression with specific stages of the estrous cycle.

\section{Expression of Vgr-1 RNA in the brain}

In addition to the expression of Vgr-1 in the meninges of the brain, Vgr-1 transcripts are detected at low levels throughout the neural tissue of newborn and adult brains. However, Vgr-1 transcripts are present at a higher level in the pyramidal layer of the hippocampus, with increased expression in the adult compared to the 16.5-day p.c. brain (data not shown).

\section{Discussion}

The observations reported here point to multiple roles for the Vgr-1 gene product in development. Vgr-1 transcripts are present at many stages of embryogenesis, in the derivatives of all three germ layers and in both the mesenchymal and epithelial components of different tissues. In addition to its potential functions in development, the presence of Vgr-1 RNA in adult tissues suggests a role in the regulation of the growth and differentiation of established systems, particularly epithelial.

It is clear from Figure 1 that the mouse oocyte contains maternally encoded Vgr-1 transcripts. Similarly, Vgl RNA is maternally encoded in Xenopus oocytes. However, in contrast to the persistence of Xenopus Vgl RNA into the gastrulation stage (Rebagliati et al. 1985), the level of Vgr-1 RNA in the mouse begins to decline in fertilized eggs and is undetectable by the two- to fourcell stage. This observation follows a general pattern of mammalian oocyte RNA turnover (Bachvarova et al. 1985 ) in which meiotic maturation results in the depletion of approximately half of the poly(A) ${ }^{+}$RNA. This loss continues rapidly through the two-cell stage (Pikó and Clegg 1982; DeLeon 1983). Substantial decreases in

Figure 2. Expression of Vgr-1 RNA in embryonic and newborn skin. Bright-field $(A, C, E, I, K, M$, and $O)$ and corresponding darkground $(B, D, F, H, I, L, N$, and $P)$ micrographs of sections hybridized with Vgr-1 antisense probe. (A) Section through dorsal skin of a day-13.5 p.c. embryo hybridized with Vgr-1 antisense probe. Bar, $100 \mu \mathrm{m}$. $(B)$ Same section as in $A$, showing that no Vgr-1 signal is seen at this stage in either the epidermis (upper arrowhead) or dermis. (C) Section through dorsal skin from a day-15.5 p.c. embryo hybridized with Vgr-1 antisense probe. (D) Same section as in $C$, showing strong signal in the suprabasal layers of the epidermis (upper arrowhead), whereas no specific signal is seen in the dermis or the hair follicles (lower arrowhead). (E) A dorsal skin section similar to $C$ taken from a different day-15.5 p.c. embryo and hybridized with TGF $\beta 2$ antisense probe. $(F)$ Same section as in $E$, showing strong signal in the dermis but no signal in the epidermis (upper arrowhead) or hair follicle (lower arrowhead). (G) Section through the upper snout of a day-16.5 p.c. embryo hybridized with Vgr-1 antisense probe. Bar, $1 \mathrm{~mm}$. $(H)$ Same section as in $G$, showing strong signal in the epidermis. No specific signal is seen elsewhere. (I) Enlarged region (upper box) from the section seen in $G$. (J) Same section as in $I$, showing abundant Vgr-1 signal to be present in the suprabasal layers of the epidermis (upper arrowhead) and absent from basal cells (lower right arrowhead), hair follicles (lower left arrowhead) and dermis. (K) Enlarged region (lower box) from the section seen in $G$. (L) Same section as in $K$, showing low Vgr-l signal confined to the uppermost layers of the lip epidermis (arrowhead). (M) Section of dorsal skin from a newborn pup hybridized with Vgr-1 antisense probe. $(N)$ Same section as in $M$, showing hybridization in the suprabasal epidermis (upper arrowhead). The basal cells, hair follicles (lower arrowheads), and dermis show no Vgr-1 signal. (O) Adjacent section to $M$ hybridized with TGF 32 sense (negative control) probe. $(P)$ Same section as in $O$, showing that no specific signal is seen in the negative control. The apparent signal at the edge of the epidermis (upper arrowhead) is due to light scattering by cornified cells. Sections were exposed for 10-20 days. (epi) Epidermis; (dm) dermis; (hf) hair follicle; (sm) smooth muscle; (bc) basal cells; (nc) nasal cavity; (pl) palate. 

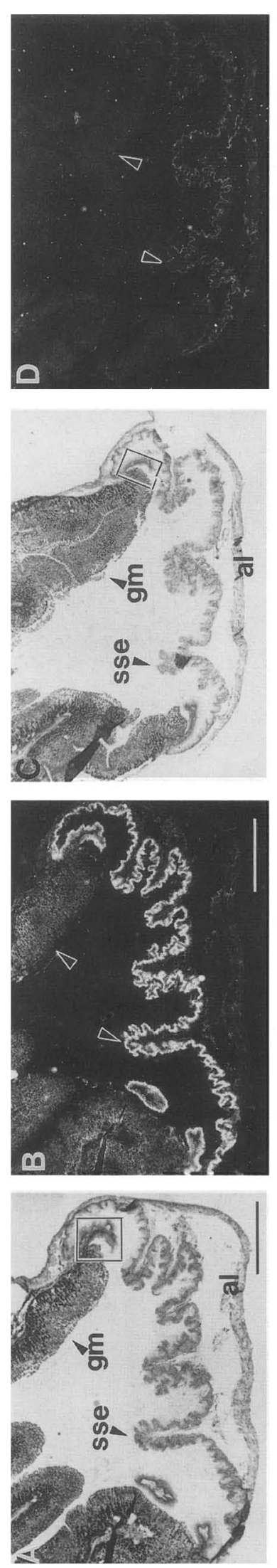

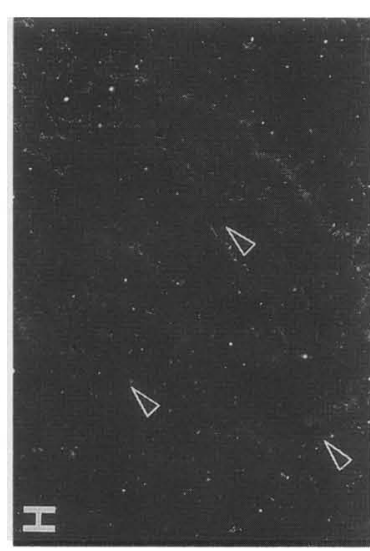

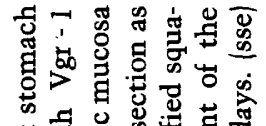

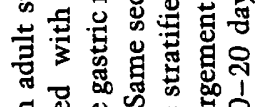

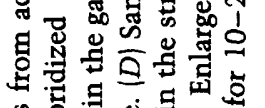

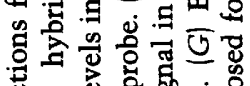

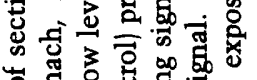

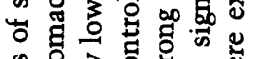

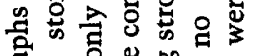

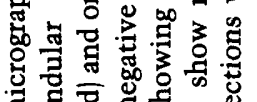

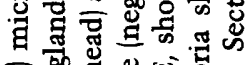

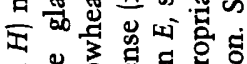

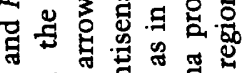
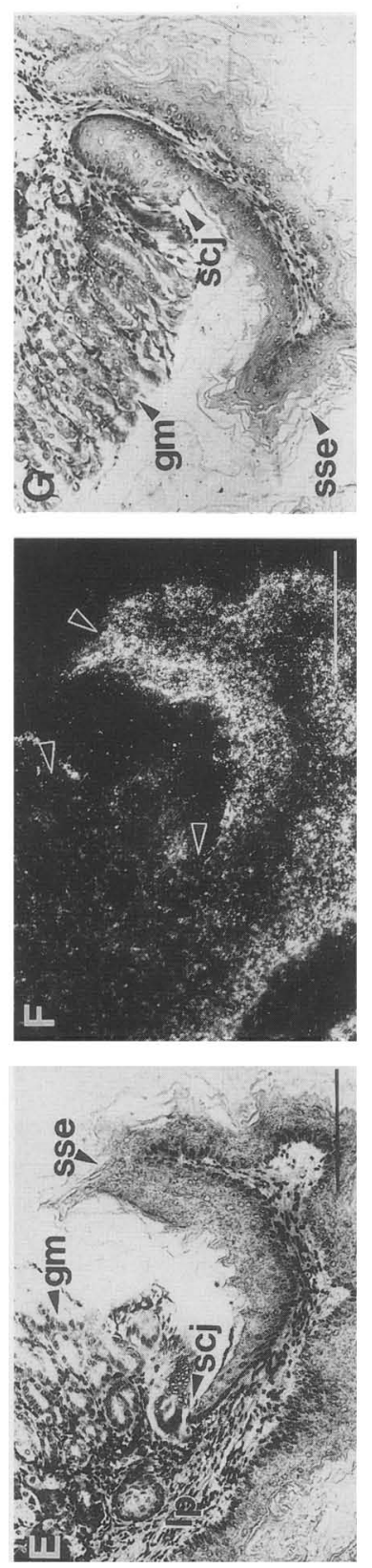

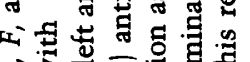

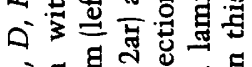

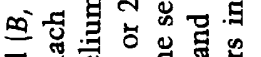

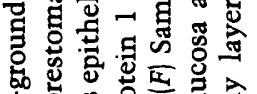

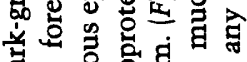

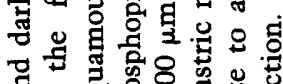

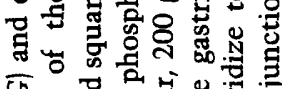

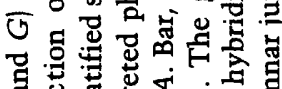

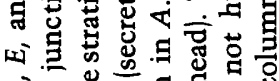

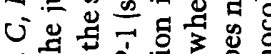

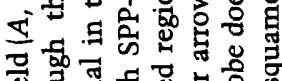

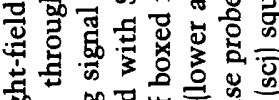
की

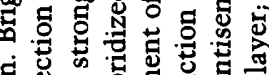

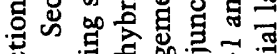

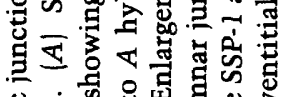

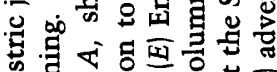

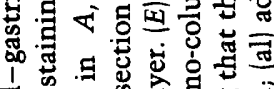

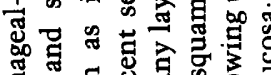

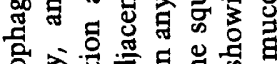

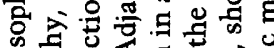

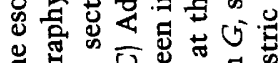

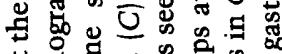

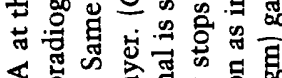

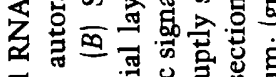

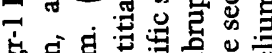

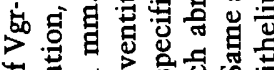

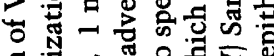

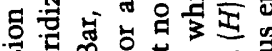

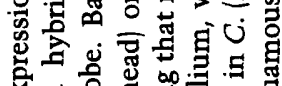

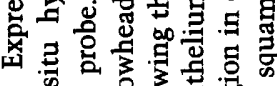
की

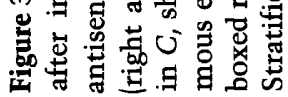



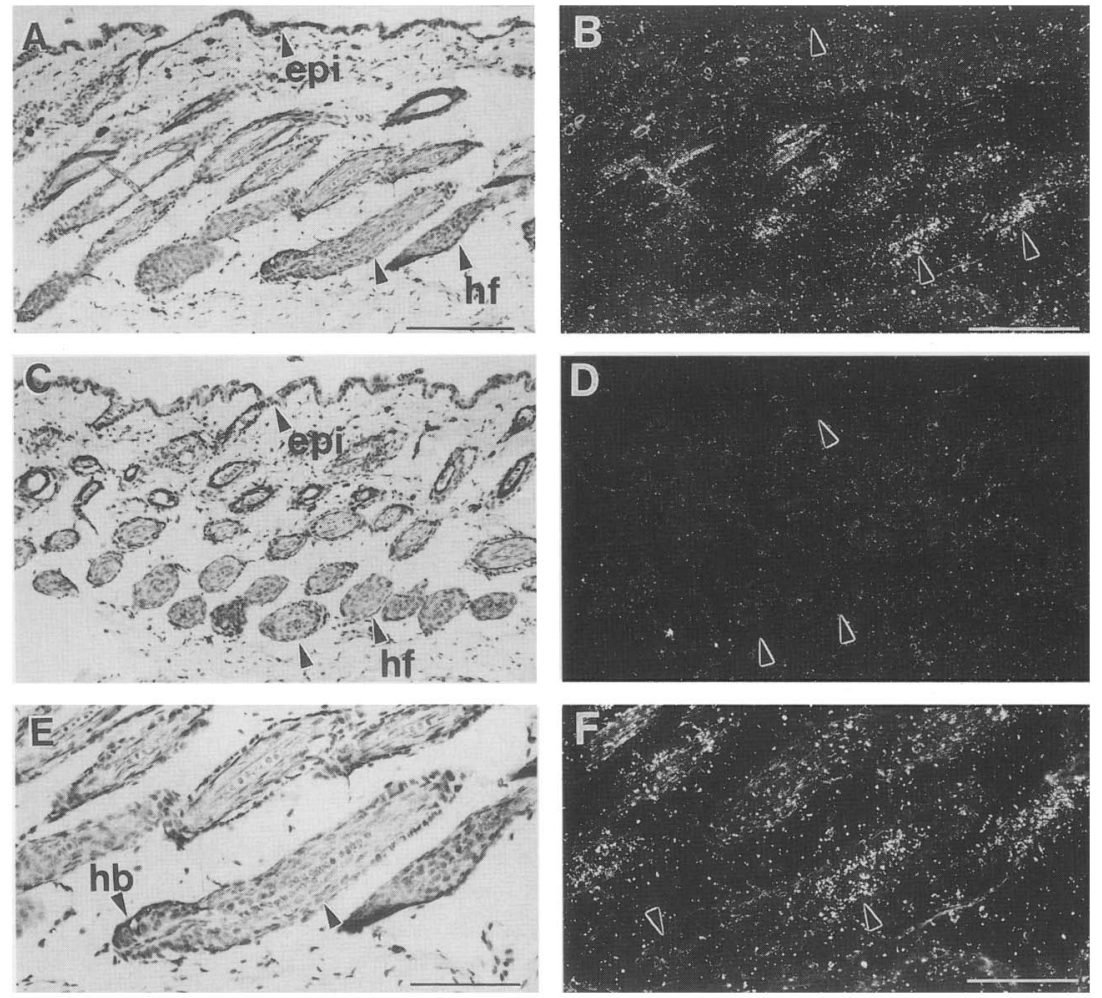

Figure 4. Expression of BMP-2a RNA in adult hair follicles. Bright-field (left) and corresponding dark-ground (right) photomicrographs of adult sections through skin after in situ hybridization, autoradiography, and staining. $(A)$ Section through adult skin with hair follicles (lower arrowheads) hybridized with BMP-2a antisense probe. Bar, $200 \mu \mathrm{m} .(B)$ Same section as in $A$, showing signal in the hair follicles. $(C)$ Adjacent section to $A$ hybridized with BMP-2a sense (control) probe. (D) Same section as in $C$, illustrating absence of signal in the hair follicles (lower arrowheads) with the control probe. (E) Same section as in $A$ at higher power. Bar, $100 \mu \mathrm{m}$. $(F)$ Same section as in $E$, showing signal in the cortex/inner root sheath of the hair follicle. Sections were exposed for 10-20 days. (epi) Epidermis; (hf) hair follicle; (hb) hair bulb.

the levels of several specific maternally encoded transcripts, including c-mos (Mutter et al. 1988) and tissuetype plasminogen activator ( $t-P A)$ (Huarte et al. 1987), by the two-cell stage have also been demonstrated. Elucidation of the role that the Vgr-1 gene product plays in oocytes and fertilized eggs must await the isolation of specific antibodies and active Vgr-1 protein, as well as the localization of specific receptors. However, it is conceivable that the Vgr-1 gene product regulates the growth and/or final maturation of female germ cells in an autocrine manner. In support of this notion, two other members of the TGF $\beta$ superfamily, TGF $\beta 1$ and MIS have been shown to promote or inhibit, respectively, oocyte meiosis (Donahoe et al. 1987; Feng et al. 1988).

In early cleavage-stage embryos, Vgr-1 RNA either is not present or is produced at levels too low to be detected by in situ hybridization, but low levels of transcripts are seen in 4.5-day p.c. blastocysts. The expression of Vgr-1 in early postimplantation embryos at a time when the three embryonic germ layers are being established is consistent with a role for the gene product in inductive tissue interactions. By analogy with the distribution of Vg-1 RNA in Xenopus embryos and the possible role of the gene product in mesoderm induction (Rebagliati et al. 1985), one might expect Vgr-1 RNA to be expressed at high levels in mouse embryos, either in the extraembryonic endoderm (hypoblast) underlying the primitive ectoderm (epiblast) or in the presumptive endoderm generated from the primitive ectoderm; however, Vgr-1 RNA is present at low levels in all identifiable layers (extraembryonic endoderm, embryonic ectoderm, and mesoderm). This observation appears to argue against a role for $\mathrm{Vgr}-1$ as a natural mesoderm inducer in early mouse embryos, although the possibility remains that the active Vgr-1 protein and/or its receptor are more localized.

In contrast to its generalized distribution in early postimplantation embryos, Vgr-1 RNA has a very localized pattern of expression in later mid-gestation embryos, newborns, and adults. A primary site of expression is in stratified squamous epithelia and a sharp discontinuity in the level of Vgr-1 RNA is seen at the junction between glandular and squamous epithelia, for example, in the adult stomach (Fig. 3). Experiments are in progress to test whether this difference in expression is regulated by signals from the underlying mesenchyme. Within stratified squamous epithelia, the level of Vgr-1 RNA appears to be related to the degree of keratinization. This is illustrated, for example, in Figure 2, G-L, where the dorsal epidermis of the upper snout contains high levels 
Lyons et al.
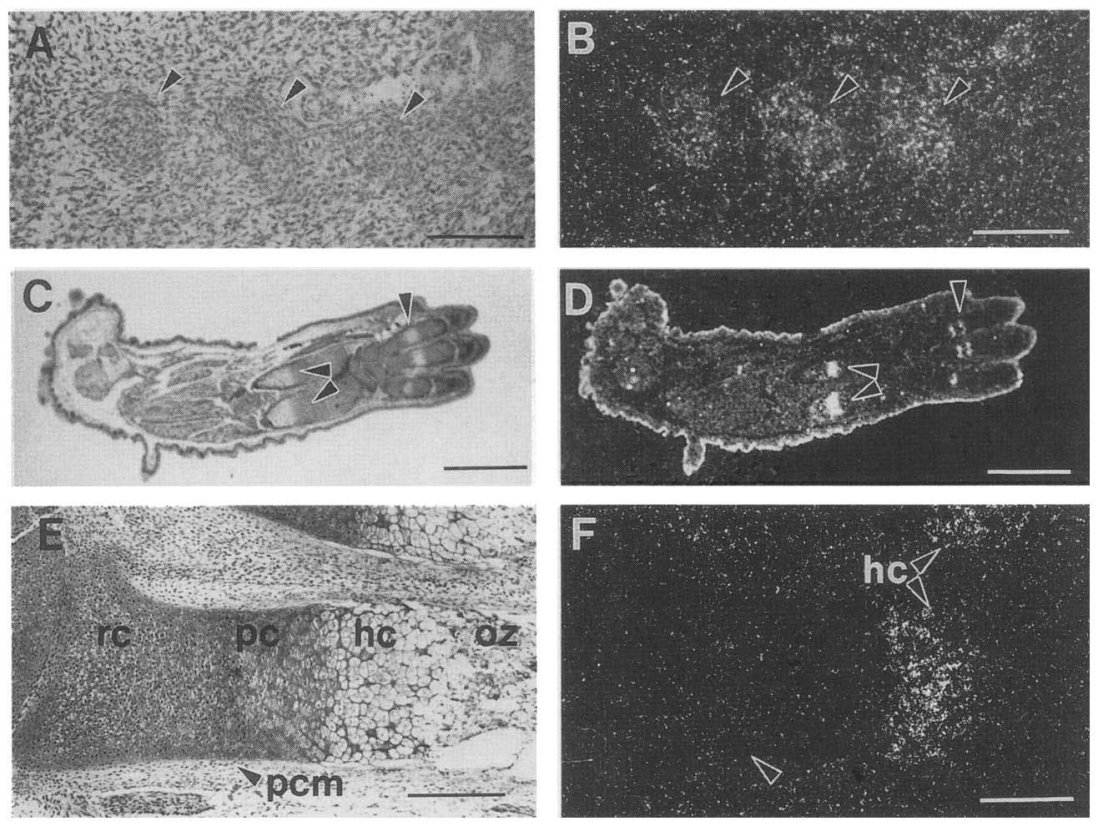

Figure 5. Expression of BMP-2a and Vgr-1 RNA in developing bone and cartilage. Bright-field (left) and corresponding dark-ground (right) photomicrographs of embryo sections after in situ hybridization, autoradiography, and staining. $(A)$ Section through developing prevertebrae (arrowheads) of a day-12.5 p.c. embryo hybridized with BMP-2a antisense probe. Bar, $150 \mu \mathrm{m}$. $(B)$ Same section as $A$, showing hybridization in the condensing mesenchyme, which will give rise to the ribs/vertebrae (arrowheads). (C) Longitudinal section through the limb of a day-15.5 p.c. embryo hybridized with BMP-2a antisense probe. Bar, $1 \mathrm{~mm}$. (D) Same section as in $C$, showing strong signal in the hypertrophic cartilage of the long bones (double arrowheads) and digits (single arrowhead). Note the strong signal in the epidermis surrounding the limb. (E) A long bone from a 17.5-day p.c. embryo, showing four stages of maturation in the developing bone. Bar, $200 \mu \mathrm{m}$. $(F)$ Same section as in $E$, showing that only the hypertrophic cartilage (double arrowheads) contains Vgr-1 RNA. The resting chondrocytes, proliferating chondrocytes, and osteogenic zone show no specific signal. Sections were exposed for 7-10 days. (rc) Resting chondrocytes; (pc) proliferating chondrocytes; (hc) hypertrophic cartilage; (oz) osteogenic zone (pcm) perichondrium.

of Vgr-1 RNA; however, the lip epithelium, which has a thicker layer of nucleated suprabasal cells and a thin stratum corneum, expresses very low levels of Vgr-1 RNA. Hybridization to the Vgr-l riboprobe is confined in the lip and palate to several highly differentiated outer layers. This correlation suggests that one function of Vgr-l may be to regulate the progression of the immediate suprabasal cells to a more differentiated and, therefore, more cornified phenotype. The observation that TGF $\beta 1$ reversibly inhibits the proliferation of human prokeratinocytes in vitro (Shipley et al. 1986) is consistent with this proposal and suggests that Vgr-1 may have some functions related to those described for TGF 1 .

Vgr-1 RNA is not confined to epithelia, as we observed high levels of expression in a variety of mesenchymal and ectomesenchymal tissues, including hypertrophic cartilage, uterine stroma, and meninges. The potential roles of Vgr-1 in the growth and differentiation of these tissues are unknown at present but are considered below. However, the expression of Vgr-1 in the meninges is particularly interesting because the Vgr-l gene has been mapped recently to a location near the congen. ital hydrocephalus (ch) locus on mouse chromosome 13 (M. Dickinson, M. Kobrin, C. Silan, D. Kingsley, M. Justice, D. Miller, J. Ceci, L. Lock, A. Lee, A. Buchberg, L. Siracusa, K. Lyons, R. Derynck, B. Hogan, N. Copeland, and N. Jenkins, in prep.). Embryos that are homozygous for the recessive $c h$ mutation die shortly after birth due, at least in part, to a failure of the subarachnoid space to develop (Grüneberg 1953; Green 1970). These mutants also have a number of other defects, including delayed growth of the long bones and cartilaginous skull. The expression of Vgr-1 transcripts in meninges and developing limbs is thus consistent with the theory that the ch mutation may be a lesion in the Vgr-1 gene. We are currently exploring this possibility.

One of the striking findings emerging from this work is that all of the members of the TGF $\beta$ superfamily that have been examined to date by in situ hybridization are expressed in developing skin and limbs in temporally and spatially distinct patterns. We note that the expression of TGF $\beta 2$ and Vgr-1 in skin coincides with the time of thickening and differentiation of the epidermis (Pelton et al. 1989; for a review of skin development, see Kopan and Fuchs 1989|. Furthermore, Lehnert and Akhurst (1988) reported that TGF $\beta 1$ transcripts are present in developing whisker follicles, and Akhurst et al. (1988) found that TGF $\beta 1$ RNA is present in the suprabasal layers of adult skin treated with the tumor promoter 12tetradecanoyl-phorbol-13-acetate (TPA). Thus, the expression of TGF $\beta 1$ and TGF $\beta 2$ in the epidermis and epithelial components of the hair follicles and Vgr-1 in the 
epidermis occurs at a time of active proliferation and differentiation, whereas the levels of transcripts for these growth factors are reduced in adult skin (Lehnert and Akhurst 1988; Pelton et al. 1989, and unpubl.|. Similarly, BMP-2a RNA expression persists in adults in the actively proliferating hair cortex cells.

A similar example of overlapping but temporally and spatially distinct patterns of expression of TGF $\beta$ superfamily members occurs in the developing limbs. The development of long bones is initiated by the condensation of the mesenchyme, proliferation and differentiation of the chondroblasts into chondrocytes, deposition of a cartilaginous matrix, hypertrophy and degeneration of the chondrocytes, calcification of cartilage, and replacement of this matrix by invading osteogenic cells, which produce a bone matrix that later becomes calcified. BMP-2a is the first member of the TGF $\beta$ superfamily known to be expressed in this sequence and is localized in condensing precartilaginous mesenchyme but is undetectable in the resting, proliferating, and differentiating chondrocytes. TGF $\beta 2$ is first detected at a later stage in the chondroblasts of the perichondrium (Pelton et al. 1989). Vgr-1 is expressed at the final stage of chondroblast differentiation in the hypertrophic cells.

This pattern raises the possibility that the expression of different members of the TGF $\beta$ superfamily is required at distinct stages of differentiation within the chondrocyte lineage to ensure the regulated progression of the condensing mesenchymal cell through to the terminally differentiated hypertrophic cartilage phenotype. The in situ hybridization evidence for this proposal is summarized in the model presented in Figure 6. We suggest that the initial expression of BMP- $2 a$ and perhaps other as yet unidentified members of the TGF $\beta$ superfamily may promote the condensation and differentiation of mesenchyme into actively proliferating chondroblasts and chondrocytes, which results in the expression of a different set of TGF $\beta$-like gene products, including TGF $\beta 2$, in the more terminally differentiated cells. The newly induced growth factors then may regulate the proliferation and differentiation of the cells in which they are produced in an autocrine manner, as well as act on additional cell types in the chondrocyte differentiation pathway in a paracrine manner to ensure a coordinated progression through this lineage. Similarly, the expression of the Vgr-1 gene in the hypertrophic cartilage may promote the terminal differentiation of these cells in an autocrine fashion, as well as affect the proliferation of the chondrocytes from which the hypertrophic cells arise by a paracrine mechanism. An additional possibility is that the Vgr-1 protein is chemotactic for the osteoblasts and osteoclasts that eventually replace the cartilaginous matrix. In our model we indicate that each of the TGF $\beta$-like gene products has autocrine and paracrine effects on proliferation and differentiation. The precise roles and sites of action in cartilage differentiation of each of these gene products will require the use of specific antibodies and purified proteins in in vivo studies; however, there is some experimental support for this model. For example, BMP-2a has been shown to induce ectopic bone formation in vivo in a process involving the migration, proliferation, and condensation of mesenchymal cells (Wozney et al. 1988). In vitro studies show that TGF $\beta 1$ and TGF $\beta 2$ can induce fetal rat muscle mesenchymal cells to undergo differentiation and synthesize cartilage-specific macromolecules in vitro (Seyedin et al. 1986, 1987). The most extensively studied protein, TGF $\beta 1$, has been shown to have autocrine and paracrine effects on the proliferation and differentiation of many of the cell types within the chondrocyte lineage in in vitro model systems (Seyedin et al. 1985; Centrella et al. 1988; Kato et al. 1988; D.M. Rosen et al. 1988). These studies thus demonstrate that TGF $\beta$-like gene products can affect growth and differentiation at many stages within the lineage, at least in vitro. Different approaches will be necessary to elucidate the specific roles each TGFß2-like gene product has in the progression of the chondrocyte lineage in vivo.

In summary, our work provides strong evidence that the Vgr-1 gene plays multiple roles in murine development and in the growth regulation of adult tissues. Furthermore, we have shown that Vgr-1 and BMP-2a, two members of the $d p p$ subgroup of the TGF $\beta$ superfamily, are expressed in temporally and spatially distinct patterns in developing skin and limbs, as are TGF $\beta 1$ and TGF $\beta 2$. This observation suggests that the coordinated expression of many TGF $\beta$-like growth factors is required during the development of specific organs.

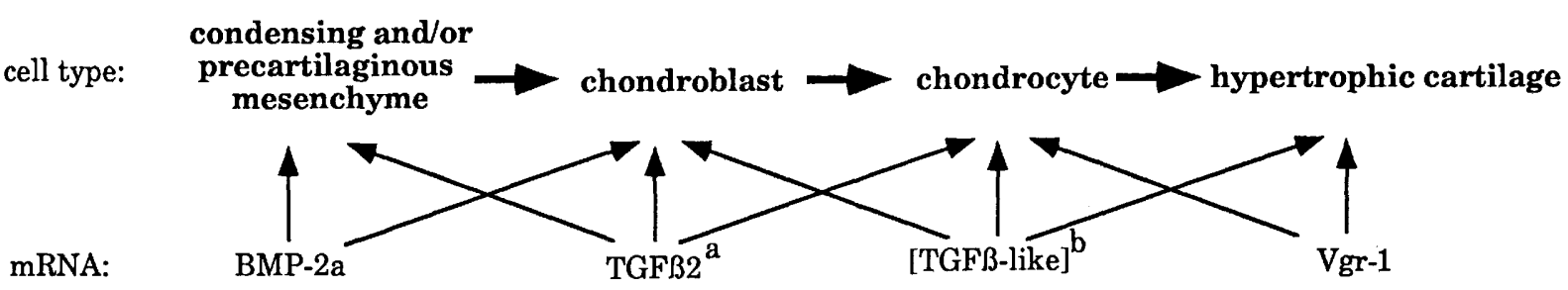

Figure 6. (Top line) The progression of cells from condensing mesenchyme through to hypertrophic cartilage; (bottom line) TGF $\beta$ like transcripts that have been reported to be expressed in each cell type. Arrows depict potential autocrine and paracrine effects of the various TGF $\beta$-like gene products. (a) Data from Pelton et al. (1989). (b) Sandberg et al. (1988) reported small amounts of TGF $\beta 1$ RNA in some chondrocytes of the growth plates and epiphyses. In contrast, Lehnert and Akhurst (1988) did not detect TGF $\beta$ RNA in the cartilage under conditions of high stringency hybridization, but they reported hybridization within the cartilage with lower stringency. This observation may thus reflect low levels of TGF $\beta 1$ RNA or the presence of a closely related member of the TGF $\beta$ superfamily. Evidence for the latter possibility is provided by Jakowlew et al. (1988), who showed that TGF $\beta 4$, which is $85 \%$ homologous to TGF $\beta 1$ at the nucleotide level, is abundant in cultured primary chick embryo chondrocytes. 


\section{Methods}

Isolation of total RNA from primary oocytes and follicle cells

For isolation of primary oocytes and follicle cells, ovaries from 12.5-gram (C57BL/6 $\times \mathrm{DBA} / 2) \mathrm{F}_{1}$ females were dissected into Dulbecco's modified Eagle's medium, containing $10 \%$ fetal bovine serum, and punctured repeatedly with a fine-gauge needle. Released primary oocytes with an intact germinal vesicle and no polar body were separated individually from follicle cells by pipetting and collected manually. Clumps of follicle cells were also collected for RNA extraction. Total RNA was isolated by disrupting cells in guanidium isothiocyanate, followed by phenol/chloroform extraction and precipitation with ethanol in the presence of $50 \mu \mathrm{g}$ of carrier tRNA, as described (Huarte et al. 1987).

\section{Preimplantation embryos}

Prepuberal $F_{1}$ females $(\sim 12.5 \mathrm{~g})$ were superovulated and mated with $F_{1}$ males as described (Hogan et al. 1986). Oviducts containing fertilized eggs and cumulus cells were fixed $\sim 21 \mathrm{hr}$ post-human chorionic gonadotropin (hCG). Cleavage-stage embryos and blastocysts were recovered from the oviduct and uterus as described (Hogan et al. 1986). For convenience of handling, they were transferred into oviducts containing fertilized one-cell eggs, and the oviducts were dissected and fixed. The fertilized eggs thus provided a positive control for the in situ hybridizations.

\section{Postimplantation embryonic and adult tissues}

Postimplantation embryos were obtained from ICR outbred females mated with $F_{1}$ males (Harlan Sprague-Dawley). Noon of the day of the vaginal plug is 0.5 days p.c.

\section{Probe construction}

An 893-bp SacI-EcoRI fragment from the $5^{\prime}$ region of the Vgr-1 cDNA (Lyons et al. 1989) was subcloned into pGEM-3Zf)-1 (Promega, Madison, Wisconsin). This fragment does not include the region of high similarity between Vgr-1 and other members of the TGF $\beta$ superfamily, thus minimizing the probability of cross hybridization. Our Vgr-1 probe shows $41 \%$ identity to the murine BMP-2a cDNA and only $37 \%$ identity to the murine TGF $\beta 1$ cDNA. The Vgr- 1 construct detects a single, $\sim 3.5-\mathrm{kb}$ transcript when hybridized to RNA by Northern analysis from all tissues except testes, where an additional 1.8 -kb transcript is detected (Lyons et al. 1989). A 1124-bp EcoRI fragment from the murine BMP-2a cDNA (M. Dickinson, M. Kobrin, C. Silan, D. Kingsley, M. Justice, D. Miller, J. Ceci, L. Lock, A. Lee, A. Buchberg, L. Siracusa, K. Lyons, R. Derynck, B. Hogan, N. Copeland, and $\mathrm{N}$. Jenkins, in prep.) was subcloned into pGEM-3Zf $(-)$ and linearized with XmaIII. This construct yields a $1-\mathrm{kb}$ riboprobe that detects a single $3.5-\mathrm{kb}$ transcript when hybridized to Northern blots (data not shown). We were unable to determine the overall identity of this construct to the closely related murine BMP- $2 \mathrm{~b}$ cDNA because the complete sequence for the murine BMP-2b cDNA was not available. However, 3 '-terminal $374 \mathrm{bp}$ of our probe is only $64 \%$ identical with the corresponding region of a partial murine BMP-2b cDNA clone. Because the remaining 620 bp of the murine BMP-2a construct encodes a region of the protein that is less highly conserved at the amino acid level (Wozney et al. 1988), we expect that the overall homology of our BMP-2a probe to the BMP-2b transcript will be $<64 \%$ at the nucleotide level. In support of this, the corresponding regions of the human BMP-2a and BMP-2b cDNAs are $58 \%$ identical. A secreted phosphoprotein 1 or $2 a$ (SPP-1)(Nomura et al. 1988) antisense riboprobe was used as a positive control in bone, uterus, and cumulus cells and as a negative control in other tissues. Single-stranded sense and antisense RNA probes were labeled with $\alpha^{-35}$ S-labeled UTP (1400 $\mathrm{Ci} / \mathrm{mm}$, New England Nuclear) to an approximate specific activity of $2 \times 10^{9} \mathrm{dpm} / \mu \mathrm{g}$ RNA, using either SP6 or T7 polymerase, essentially as described by Hogan et al. (1986). These probes were reduced to an average size of $100-150$ bp by limited alkaline hydrolysis (Cox et al. 1984) and used for hybridization at a final concentration of $2 \times 10^{5} \mathrm{dpm} / \mu \mathrm{l}$.

\section{Northern hybridization}

Total RNA from undifferentiated F9 cells and uteri of 9.5-day p.c. pregnant mice was isolated as described (Lyons et al. 1989). Northern hybridizations on $20 \mu \mathrm{g}$ of total RNA or $\sim 150$ primary oocytes were carried out as described in Lyons et al. (1989).

\section{In situ hybridization}

In situ hybridizations and washes were carried out at high stringency, essentially as described by Nomura et al. (1988), with the following modifications. Sections were hybridized for $14-16 \mathrm{hr}$ at $50^{\circ} \mathrm{C}$ in $50 \%$ formamide. Following hybridization, the sections were washed for $1 \mathrm{hr}$ in $50 \%$ formamide at $50^{\circ} \mathrm{C}$, treated with RNase A (Sigma) at a concentration of $10 \mu \mathrm{g} / \mathrm{ml}$ for $20 \mathrm{~min}$ at $37^{\circ} \mathrm{C}$, and washed at $55^{\circ} \mathrm{C}$ for $1 \mathrm{hr}$ in $2 \times \mathrm{SSC}$ and $1 \mathrm{hr}$ in $0.1 \times$ SSC. Exposure times were between 1 and 3 weeks, with Ilford $\mathrm{K} 5$ emulsion. After development, the slides were stained with $0.2 \%$ toluidine blue and analyzed on a Zeiss Axiophot microscope using bright-field and dark-ground optics. The slides were photographed using Kodak Technical Pan film.

\section{In situ analysis of grain density}

Autoradiographic silver grains in slides developed after 10-20 days of exposure were counted on photographs of dark-ground images. Grain density was measured without subtraction of background grain counts. Densities over five fertilized eggs were calculated as percent of the grain density observed in two primary oocytes from the same set of slides.

\section{Acknowledgments}

We thank Sofie Hashmi for excellent technical assistance, Rik Derynck for providing the BMP-2a cDNA clone, and Lynn Matrisian, Hal Moses, Jeff Holt, and Ed Yang for helpful comments on the manuscript. K.M.L. was supported by National Cancer Institute postdoctoral training grant 5T32-CA0959292. R.W.P. was supported by National Institutes of Health grant T32GM07347.

\section{References}

Akhurst, R.J., F. Fee, and A. Balmain. 1988. Localized production of TGF $\beta$ mRNA in tumour promoter-stimulated mouse epidermis. Nature 331: 363-365.

Bachvarova, R., V. DeLeon, A. Johnson, G. Kaplan, and B.V. Paynton. 1985. Changes in total RNA, polyadenylated RNA, and actin mRNA during meiotic maturation of mouse oocytes. Dev. Biol. 108: 325-332. 
Centrella, M., T.L. McCarthy, and E. Canalis. 1988. Skeletal tissue and transforming growth factor $\beta$. FASEB J. 2: 30063073.

Coffey, R.J., C.C. Bascom, N.J. Sipes, R. Graves-Deal, B.E. Weissman, and H.L. Moses. 1988. Selective inhibition of growth-related gene expression in murine keratinocytes by transforming growth factor $\beta$. Mol. Cell. Biol. 8: 3088-3093.

Cox, K.H., D.V. DeLeon, L.M. Angerer, and R.C. Angerer. 1984. Detection of mRNAs in sea urchin embryos by in situ hybridization using asymmetric RNA probes. Dev. Biol. 101: 485-502.

Dale, L. and M.W. Slack. 1987. Regional specification within the mesoderm of early embryos of Xenopus laevis. Development 100: 279-295.

Daniel, C.W., G.B. Silberstein, K. Van Horn, P. Strickland, and S. Robinson. 1989. TGF-beta-1-induced inhibition of mouse mammary ductal growth: developmental specificity and characterization. Dev. Biol. 135: 20-30.

DeLeon, V., A. Johnson, and R. Bachvarova. 1983. Half-lives and relative amounts of stored and polysomal ribosomes and poly $(\mathrm{A})^{+}$RNA in mouse oocytes. Dev. Biol. 98: 400-408.

Donahoe, P.K., R.L. Cate, D.T. MacLaughlin, J. Epstein, A.F. Fuller, M. Takahashi, J.P. Coughlin, E.G. Ninfa, and L.A. Taylor. 1987. Müllerian inhibiting substance: Gene structure and mechanism of action of a fetal regressor. Recent Prog. Horm. Res. 43: 431-467.

Edwards, D.R., G. Murphy, J.J. Reynolds, S.E. Whitham, A.J.P. Docherty, P. Angel, and J.K. Heath. 1987. Transforming growth factor beta modulates the expression of collagenase and metalloproteinase inhibitor. EMBO I. 6: 1899-1904.

Feng, P., K.J. Catt, and M. Knecht. 1988. Transforming growth factor $\beta$ stimulates meiotic maturation of the rat oocyte. Endocrinology 122: 181-186.

Flanders, K.C., N.L. Thompson, D.S. Cissel, E. Van ObberghenSchilling, C.C. Baker, M.E. Kass, R.L. Ellingsworth, A.B. Roberts, and M.B. Sporn. 1989. Transforming growth factor$\beta 1$ : Histochemical localization with antibodies to different epitopes. J. Cell Biol. 108: 653-660.

Green, M. 1970. The developmental effects of congenital hydrocephalus (ch) in the mouse. Dev. Biol. 23: 585-608.

Grüneberg, H. 1953. Genetical studies on the skeleton of the mouse: VII. Congenital hydrocephalus. J. Genet. 51: $327-$ 358.

Heine, U.I., E.F. Munoz, K.C. Flanders, L.R. Ellingsworth, H.-Y.P. Lam, N.L. Thompson, A.B. Roberts, and M.B. Sporn. 1987. Role of transforming growth factor- $\beta$ in the development of the mouse embryo. I. Cell. Biol. 105: 2861-2876.

Hogan, B.L.M., F. Constantini, and E. Lacy. 1986. Manipulating the mouse embryo. A laboratory manual. Cold Spring Harbor Laboratory, Cold Spring Harbor, New York.

Huarte, J., D. Belin, A. Vassalli, S. Strickland, and J.-D. Vassalli. 1987. Meiotic maturation of mouse oocytes triggers the translation and polyadenylation of dormant tissue-type plasminogen activator mRNA. Genes Dev. 1: 1201-1211.

Ignotz, R.A. and J. Massagué. 1986. Transforming growth factor- $\beta$ stimulates the expression of fibronectin and collagen and their incorporation into the extracellular matrix. J. Biol. Chem. 261: 4337-4345.

Irish, V.G. and W.M. Gelbart. 1987. The decapentaplegic gene is required for dorsal-ventral patterning of the Drosophila embryo. Genes Dev. 1: 868-879.

Jakowlew, S.B., P.J. Dillard, M.B. Sporn, and A.B. Roberts. 1988. Complementary deoxyribonucleic acid cloning of a messenger ribonucleic acid encoding transforming growth factor $\beta 4$ from chick embryo chondrocytes. Mol. Endocrinol. 2: $1186-1195$
Kato, Y., I. Masahiro, T. Koike, F. Suzuki, and Y. Takano. 1988. Terminal differentiation and calcification in rabbit chondrocyte cultures grown in centrifuge tubes: Regulation by transforming growth factor $\beta$ and serum factors. Proc. Natl. Acad. Sci. 85: 9552-9556.

Kimmelman, D. and M. Kirschner. 1987. Synergistic induction of mesoderm by FGF and TGF- $\beta$ and the identification of an mRNA coding form FGF in the early Xenopus embryo. Cell 51: 869-877.

Koplan, R. and E. Fuchs. 1989. A new look into an old problem: Keratins as tools to investigate determination, morphogenesis, and differentiation in skin. Genes Dev. 3: 1-15.

Lehnert, S.A. and R.J. Akhurst. 1988. Embryonic expression pattern of TGF beta type-1 RNA suggest both paracrine and autocrine mechanisms of action. Development 104: 263273.

Lund, L.R., A. Riccio, P.A. Andreasen, L.S. Nielsen, P. Kristensen, M. Laiho, O. Saksela, F. Blasi, and K. Dan $\varnothing .1987$. Transforming growth factor- $\beta$ is a strong and fast acting positive regulator of the level of type-1 plasminogen activator inhibitor mRNA in WI-38 human lung fibroblasts. EMBO $J$. 6: $1281-1286$.

Lyons, K.M. and B.L.M. Hogan. 1990. TGFß-like genes in mammalian development. In Proceedings of the 48th Symposium of the Society for Developmental Biology. (ed. W.F. Loomis) Alan R. Liss, New York. (In press).

Lyons, K.M., J.L. Graycar, A. Lee, S. Hashmi, P.B. Lindquist, E.Y. Chen, B.L.M. Hogan, and R. Derynck. 1989. Vgr-1, a mammalian gene related to Xenopus $\mathrm{Vg}-1$ and a new member of the transforming growth factor $\beta$ gene superfamily. Proc. Natl. Acad. Sci. 86: 4554-4558.

Moses. H.L., R.F. Tucker, E.B. Leof, R.J. Coffey, J. Halper, and G.D. Shipley. 1985. Type beta transforming growth factor is a growth stimulator and a growth inhibitor. Cancer Cells 3: $65-71$.

Mutter, G.L., G.S. Grills, and D.J. Wolgemuth. 1988. Evidence for the involvement of the proto-oncogene c-mos in mammalian meiotic maturation and possibly very early embryogenesis. $E M B O$ I. 7: 683-689.

Nomura, S., A.J. Wills, D.R. Edwards, J.K. Heath, and B.L.M. Hogan. 1988. Developmental expression of 2ar (osteopontin) and SPARC (osteonectin) RNA as revealed by in situ hybridization. J. Cell. Biol. 106: 441-450.

Pelton, R.W., S. Nomura, H.L. Moses, and B.L.M. Hogan. 1989. Expression of transforming growth factor $\beta 2$ RNA during murine embryogenesis. Development 106: 759-767.

Pikó, L. and K.B. Clegg. 1982. Quantitative changes in total RNA, total poly(A), and ribosomes in early mouse embryos. Dev. Biol. 89: 362-378.

Rebagliati, M.R., D.L. Weeks, R.P. Harvey, and D.A. Melton. 1985. Identification and cloning of localized maternal RNAs from Xenopus eggs. Cell 42: 769-777.

Roberts, A.B., M.A. Anzano, L.M. Wakefield, N.S. Roche, D.F. Stern, and M.B. Sporn. 1985. Type $\beta$ transforming growth factor: A bifunctional regulator of cellular growth. Proc. Natl. Acad. Sci. 82: 119-123.

Roberts, A.B., M.B. Sporn, R.K. Assoian, J.M. Smith, N.S. Roche. L.M. Wakefield, U.I. Heine, L.A. Liotta, V. Falanga, J.H. Kehrl, and A.S. Fauci. 1986. Transforming growth factor type $\beta$ : Rapid induction of fibrosis and angiogenesis in vivo and stimulation of collagen formation in vitro. Proc. Natl. Acad. Sci. 83: 4167-4171.

Rosa, F., A.B. Roberts, D. Danielpour, L.L. Dart, M.B. Sporn, and I.B. Dawid. 1988. Mesoderm induction in amphibians: The role of TGFß2-like factors. Science 239: 783-785.

Rosen, D.M., S.A. Stempien, A.Y. Thompson, and S.M. 
Seyedin. 1988. Transforming growth factor-beta modulates expression of osteoblast and chondroblast phenotypes in vitro. J. Cell Physiol. 134: 337-346.

Rosen, V., E.A. Wang, J.M. Wozney, P. Cordes, D. McQuaid, and L. Kurtzberg. 1988. Purification and molecular cloning of a novel group of BMPs and localization of BMP mRNAs in developing bone. Third Int. Conf. on the Chemistry and Biology of Mineralized Tissues. p. 76.

Russell, W.E., R.J. Coffey, A.J. Ouellette, and H.L. Moses. 1988. Type $\beta$ transforming growth factor reversibly inhibits the early proliferative response to partial hepatectomy in the rat. Proc. Natl. Acad. Sci. 85: 5126-5130.

St. Johnston, R.D. and W. M. Gelbart. 1987. Decapentaplegic transcripts are localized along the dorsal-ventral axis of the Drosophila embryo. EMBO I. 6: 2785-2791.

Sandberg, M., T. Vuorio, H. Hirvonen, K. Alitalo, and E. Vuorio. 1988. Enhanced expression of TGF- $\beta$ and $c-f o s$ mRNAs in the growth plates of developing human long bones. Development 102: 461-470.

Seyedin, S.A., T.C. Thomas, A.Y. Thompson, D.M. Rosen, and K. A. Piez. 1985. Purification and characterization of two cartilage-inducing factors from bovine demineralized tissue. Proc. Natl. Acad. Sci. 82: 2267-2271.

Seyedin, S.J., P.R. Segarini, D.M. Rosen, A.Y. Thompson, H. Bentz, and J. Graycar. 1987. Cartilage-inducing factor-B is a unique protein structurally and functionally related to transforming growth factor- $\beta$. J. Biol. Chem. 262: 19461949.

Seyedin, S.M., A.Y. Thompson, H. Bentz, D.M. Rozen, I.M. McPherson, A. Conit, N.R. Siegel, G.R. Gallupi, and K.A. Piez. 1986. Cartilage-inducing factor-A. I. Biol. Chem. 261: 5693-5695.

Shipley, G.D., M.R. Pittelkow, J.J. Wille, R.E. Scott, and H.L. Moses. 1986. Reversible inhibition of normal human prokeratinocyte proliferation by type $\beta$ transforming growth factor-growth inhibitor in serum-free medium. Cancer Res. 46: $2068-2071$.

Silberstein, G.B. and C.W. Daniel. 1987. Reversible inhibition of mammary gland growth by transforming growth factor $\beta$. Science 237: 291-293.

Smith, J.D. 1989. Mesoderm induction and mesoderm-inducing factors in early amphibian development. Development 105: $665-677$.

Thompson, N.L., K.C. Flanders, J.M. Smith, L.R. Ellingsworth, A.B. Roberts, and M.B. Sporn. 1989. Expression of transforming growth factor- $\beta 1$ in specific cells and tissues of adult and neonatal mice. $J$. Cell Biol. 108: 661-669.

Wozney, J.M., V. Rosen, A.J. Celeste, L.M. Mitsock, M.J. Whitters, R.W. Kriz, R.M. Hewick, and E.A. Wang. 1988. Novel regulators of bone formation: Molecular clones and activities. Science 242: 1528-1534. 


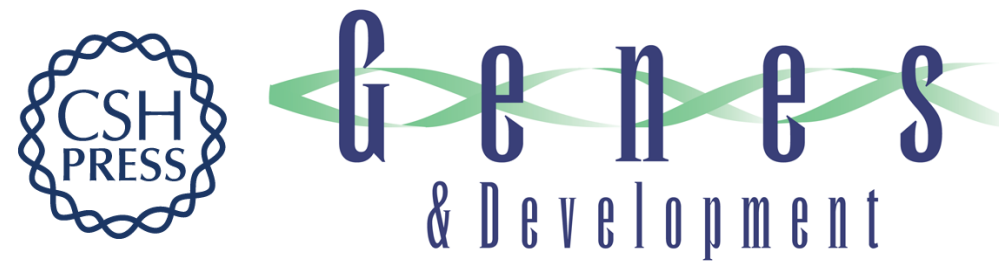

\section{Patterns of expression of murine Vgr-1 and BMP-2a RNA suggest that transforming growth factor-beta-like genes coordinately regulate aspects of embryonic development.}

K M Lyons, R W Pelton and B L Hogan

Genes Dev. 1989, 3:

Access the most recent version at doi:10.1101/gad.3.11.1657

References This article cites 45 articles, 25 of which can be accessed free at:

http://genesdev.cshlp.org/content/3/11/1657.full.html\#ref-list-1

License

Email Alerting

Service

Receive free email alerts when new articles cite this article - sign up in the box at the top right corner of the article or click here.

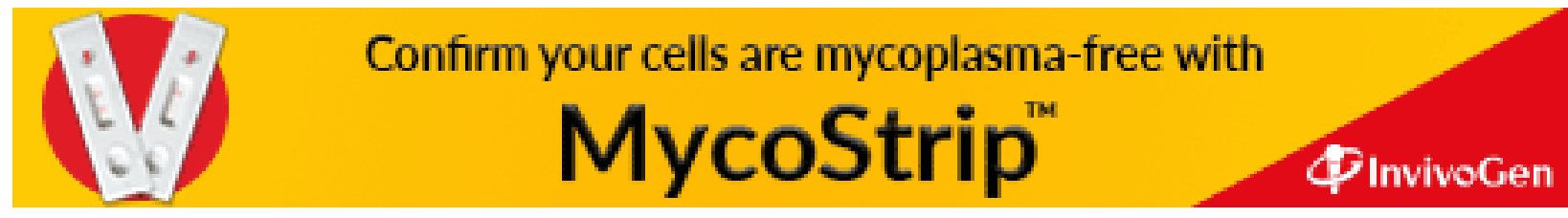

